\title{
A Comparative Analysis of Single Umbilical Tangential Incision vs. Conventional Three-port Totally Extraperitoneal Inguinal Hernia Repair
}

\author{
Hyun Su Choi, M.D., Ji Young Sul, M.D., Ph.D. \\ Department of Surgery, Chungnam National University School of Medicine, Daejeon, Korea
}

\begin{abstract}
Purpose: We have explored the question of what benefits SUTI-TEP can provide over conventional three-port TEP (C-TEP) surgery for the treatment of inguinal hernia.

Methods: One hundred forty cases (70 SUTI-TEP and 70 C-TEP) were reviewed in this study. SUTITEP procedure was carried out with SILS ${ }^{\mathrm{TM}}$ port as we reported before. Patient demographics and perioperative outcomes of SUTI-TEP were analyzed and compared with those of C-TEP.

Results: There was no conversion to open surgery or C-TEP in SUTI-TEP group. Median VAS immediate postoperatively was slightly higher in SUTI-TEP group, but it was statistically significant. POD $7^{\text {th }}$ day pain after surgery were similar in both groups. Regarding the length of operation time, SUTI-TEP group (71.2 min) was significantly longer than that of the C-TEP group (41 min) $(p<0.001)$. There was no major morbidity or mortality postoperatively in either group. There was also no recurrence or chronic pain during follow up. Patient overall satisfaction including cosmetic outcome was excellent in SUTI-TEP group.

Conclusion: Although the longer operation time tends to be required, SUTI-TEP can provide better cosmesis and patient satisfaction, and also safety. That may be good indicator of the success of after surgery. Also we should not presume that one large incision causes less pain than small multiple incision because it is just single one. If there is more pain in one single wound, we should find the solution through the innovation of technology or preventive measures.
\end{abstract}

Received September 26, 2016

Revised October 24, 2016

Accepted October 26, 2016

Corresponding author

Ji Young Sul

Department of Surgery, Chungnam

National University School of

Medicine, 282 Munhwa-ro, Jung-gu,

Daejeon 35015, Korea

Tel: +82-42-280-7175

Fax: +82-42-257-8024

E-mail: jysul@cnu.ac.kr

Keywords: Hernia, Inguinal/surgery, Laparoscopy/methods, Herniorrhaphy/methods, Treatment outcome

This is an Open Access article distributed under the terms of the Creative Commons Attribution Non-Commercial License (http:// creativecommons.org/licenses/by-nc/4.0/) which permits unrestricted non-commercial use, distribution, and reproduction in any medium, provided the original work is properly cited.

Copyright (C) 2017 The Journal of Minimally Invasive Surgery. All rights reserved.

\section{INTRODUCTION}

Single incision laparoscopic surgery (SILS) has been gaining interest because of several theoretical advantages over conventional laparoscopic surgery for certain kind of operations: reduced surgical trauma due to reduced number of incisions, less pain after surgery, fewer trocar related complications, faster recovery and early return to daily life, and better $\cos ^{-}$ metic results.
However, despite its apparent popularity of SILS, the application of SILS to inguinal hernia surgery is still limited and SILS-TEP has even more limited application. ${ }^{1}$ This is because small extraperitoneal working space enhances technical challenges of SILS, and the resultant scar is so relatively large that it can be visible after surgery.

The theoretical benefits of SILS are obvious, but it's unclear whether the benefits of SILS-TEP procedure really exist.

The aim of this study is to investigate whether SILS ingui- 
nal hernia repair technique, author's SUTI-TEP procedure, can provide favorable advantage over conventional three-port laparoscopic TEP surgery for the treatment of inguinal hernia.

\section{MATERIALS AND METHODS}

The study group comprised 140 patients who underwent primary, unilateral inguinal hernia repair from Aug 2013 to Mar 2016. The 140 patients were divided into two groups. The SUTI-TEP group consisted of 70 patients who underwent single umbilical tangential incision TEP inguinal hernia repair $^{2}$ and the C-TEP group consisted of matched number of 70 patients who underwent conventional three-port TEP repair. Only primary unilateral inguinal hernias were taken into account in this study because the number of bilateral or recurrent inguinal hernias was not enough for the comparison of outcomes for both groups.

All operations were performed by one surgeon who has passed learning curve and specializes in laparoscopic hernia repair including multi- and single port surgery. SUTI-TEP procedure was carried out with author's umbilical tangential skin incision and of SILS ${ }^{\mathrm{TM}}$ port (Medtronic, Minneapolis, Minnesota, USA) as we reported before. ${ }^{2}$

Conventional TEP procedures was performed using a single $15 \mathrm{~mm}$ infra- or subumbilical incision and two other $5 \mathrm{~mm}$ incisions in the midlines and the rest of procedure was same as reported before. ${ }^{3-6}$

In addition to patient demographic data, we recorded perioperative outcomes (operative time, length of hospital stay, postoperative pain, postoperative complications, chronic pain, and recurrence). The postoperative pain score was assessed using the Visual Analog Scale (VAS), ranging from 0 to 10, on the first and seventh day postoperative. At three months we recorded overall patient satisfaction including cosmetic outcome through interviews with all patients. Patient satisfaction rates are used to assess the success of interventions we asked

Table 1. Patient demographics

\begin{tabular}{|lccc|}
\hline & C-TEP & SUTI-TEP & $p$ value \\
\hline No. of cases & 70 & 70 & \\
\hline Age (ranges) & $61.9(25 \sim 83)$ & $58.2(21 \sim 79)$ & 0.151 \\
\hline Gender (M:F) & $63: 7$ & $60: 10$ & 0.441 \\
\hline BMI (kg/m²) & $25.8 \pm 2.38$ & $26.6 \pm 3.2$ & 0.310 \\
\hline Hernia side (R:L) & $35: 35$ & $42: 28$ & \\
\hline Hernia type (D:I:I:F:P) & $21: 43: 2: 4$ & $15: 49: 0: 6$ & \\
\hline
\end{tabular}

TEP $=$ Totally Extraperitoneal; SUTI $=$ Single Umbilical Tangential Incision; $\mathrm{D}=$ direct; $\mathrm{I}=$ indirect; $\mathrm{F}=$ femoral $; \mathrm{P}=$ pantaloon hernia. the patient to rate their overall satisfaction on a five-point scale (on a scale of 1 to $5,5=$ best satisfaction, $1=$ no satisfac tion). After putting all three factors together; (1) meeting preoperative expectations (cosmetic outcome) (2) satisfaction with pain relief (3) satisfaction with hospital environment?

Patient demographics and perioperative outcomes of SUTITEP were analyzed and compared with those of C-TEP.

All statistical analyses were conducted by using SPSS ver. 22.0 (Chicago, IL, USA). The Student's t-test for continuous variables and chi-square and Fisher's exact tests for categorical variables were used, and the level of significance was set at 0.05 .

\section{RESULTS}

The demographic characteristics were similar in the two groups (Table 1). In SUTI-TEP group, no procedure was converted to open or C-TEP repair. There was no significant difference in postoperative hospital stay or complication rate (Table 2).

Complications occurred in 10 cases in C-TEP group and 9 cases in SUTI-TEP group (not significant). All of them were minor complications: wound seroma or urinary retention. No major complications occurred (Table 2).

The immediate postoperative VAS was slightly higher and significant statistically in the SUIT-TEP than in the C-TEP group (2.7 vs. 3.2). The VAS on postoperative day 7 was com-

Table 2. Operative data

\begin{tabular}{lccc}
\hline & C-TEP & SUTI-TEP & $p$ value \\
\hline Operation times (ranges) & 41 & 71.2 & 0.000 \\
& $(35 \sim 70)$ & $(60 \sim 120)$ & \\
Hospital Stay (postoperative day) & 1 & 1 & \\
\hline VAS immediate postop & 2.7 & 3.2 & 0.007 \\
VAS post op $7^{\text {th }}$ day & 1.5 & 1.7 & 0.42 \\
\hline Postop complications (\%) & $10(14.2)$ & $9(12.8)$ & \\
Wound seroma & $3(4.2)$ & $3(4.2)$ & 0.319 \\
Urinary retention & $7(10.0)$ & $6(8.5)$ & 0.219 \\
\hline Recurrence & 0 & 0 & \\
Conversion to & & & \\
Open & 0 & 0 & \\
C-TEP & 0 & 0 & \\
Chronic pain & 0 & 0 & \\
Patient satisfaction & $3.35 \pm 1.1$ & $4.4 \pm 1.1$ & 0.000 \\
\hline Postop follow-up (months) & $5 \sim 36$ \\
\hline
\end{tabular}

TEP $=$ Totally Extraperitoneal; SUTI = Single Umbilical Tangential Incision. 
parable in both groups (Table 2).

The mean postoperative hospital stay was all 1 day. They all discharged on the day after the operation (Table 2).

Mean operative time was significantly longer in the SUTITEP group (41 vs 71.2). There was no recurrence in both groups during the follow up period. No significant chronic pain occurred in both groups. Patient satisfaction including wound cosmetic effects was significantly better in the SUTITEP group (Table 2).

\section{DISCUSSION}

The potential benefits of treatment must outweigh the potential risks and harm to the patients. So clinical success has been measured by lack of complications or by specific clinical parameters: postoperative complications, rate of recurrence, and the incidence of chronic pain. Also patient satisfaction is perhaps one of the most important criterion of success, especially in service industries.

The potential advantage of SUTI is its reduction in the number of skin incisions, which in turn might translate into less postoperative pain, a shorter convalescence, ${ }^{3}$ and also a good wound cosmesis. ${ }^{4}$

The safety and feasibility outcome results from a current study, which is designed to compare SUTI-TEP and C-TEP procedures, have shown no differences in length of hospital stay, POD $7^{\text {th }}$ pain VAS score, postoperative complications, and chronic pain just like as demonstrated by other papers. ${ }^{5,6}$ No recurrence was noted during follow up (5 36 months) in both groups.

It was no surprise that the mean operative time was longer in SUTI-TEP than in C-TEP. SUTI has technical limitations, such as loss of triangulation and reduced freedom of instruments, surgeon's ergonomics, and surgical exposure, ${ }^{8}$ which make SUTI-TEP more demanding procedure. Ambidexterity through increasing exposure to the technique and future improvements in instruments including robots may help reduce operating times.

With author's previously reported modification of umbilical skin incision and trocar dimension, SUTI-TEP can be done safely and successfully. The cosmetic effect of SUTI-TEP was excellent and most of the time it showed almost scarless effect. Patient satisfaction was so high that the patient would rather choose SUTI-TEP if the result of other outcomes are the same in both groups. So what benefits SUTI-TEP can provide to the patient over conventional laparoscopic surgery surgery for the treatment of inguinal hernia? "Same Safety, Better Cosmesis and Patient Satisfaction". They might be good indicator of success after surgery.

Another important issue that we would like to bring up in
SILS-TEP is a topic or a hidden problem that surgeons haven't talked much about. Some of the potential advantages of SILS technique are reduced number of incisions, reduced surgical trauma, so less pain after surgery. Is it really less painful postoperatively after SILS through the umbilicus?

Some worries about SILS surgery include the possible increase in pain because of larger fascial incisions needed to place the large ports into the abdomen and increased risk of umbilical hernia formation. ${ }^{9}$

But there is still a discrepancy in reports over immediate postoperative pain. Some reports showed comparable postoperative pain, and other reports didn't mention about pain between two groups. ${ }^{1,5,6,10}$ In our study and some other researchers described that patients suffered more pain on immediate postoperative day. ${ }^{1,3}$

In our SUTI-TEP experience of using many different kinds of SILS port including home-made glove port, it seemed that patients suffered more immediate postoperative pain after SILS than after conventional multi-port surgery (data not shown). In our previous study using SILS ${ }^{\mathrm{TM}}$ port (Medtronic, Minneapolis, Minnesota, USA), there was also statistical significance of immediate postoperative VAS score between two groups, ${ }^{1}$ but not significant in POD 7. Also in this study immediate postoperative pain was actually slightly more according to patienttoperative pain after SILS than after conventional multi-port surgery (data not shown)AS) difference was not that big for both (conventional vs. SILS) groups. This is because pain is a complex and subjective experience that poses a number of measurement challenges. ${ }^{11}$ and pain scale is not completely accurate and reliable in assessing pain level. ${ }^{12}$

Postoperative pain is related not only to the size of the incision but also to other several factors, such as extent of dissection, wound compression by instruments, variability of individual pain threshold, suture techniques, the operative time, and sensory nerve endings in the umbilicus skin etc. The relatively dense sensory innervation in the female umbilical skin was reported. ${ }^{13}$

Also, the shape of the trocar might be one among several factors that may cause more postoperative pain SILS-TEP. Laparoscopic TEP surgery is done in the extraperitoneal space, but all SILS ports on the market are not made for that purpose, but made for the use of intra-abdominal access. This could raise an issue of how SILS-TEP trocar should be designed. The TEP trocar should have a specific three-dimensional shape that fits into the structurally-specific retrorectus space.

So whether patients choose or demand SILS-TEP or not is determined not by just wound size or cosmetic effect but by several factors affecting patient satisfaction. If the patient satisfy, then the operation will be demanded. The SILS technique 
once labeled as "only interested in cosmesis" is now the next step in the progression of laparoscopic surgery.

Author's umbilical skin incision technique (SUTI-TEP) has the benefits of excellent wound cosmesis, with which the scar can be hidden within the natural crease of the umbilicus. But its value is not just only in cosmetic outcome: its worth comes from overall patient satisfaction embracing all clinical parameters.

Also we should not presume that one large incision causes less pain than small multiple incision because it is just single one. If there is more pain in one single wound, we should find the solution through the innovation of technology or preventive measures.

\section{REFERENCES}

1) Tran H. Safety and efficacy of single incision laparoscopic surgery for total extraperitoneal inguinal hernia repair. Jsls 2011;15:47-52.

2) Lim MH, Sul JY. SUTI (Single Umbilical Tangential Incision) Laparoscopic TEP Inguinal Hernia Repair. J Minim Invasive Surg 2015;18:24-29.

3) Deveci U, Barbaros U, Kapakli MS, et al. The comparison of single incision laparoscopic cholecystectomy and three port laparoscopic cholecystectomy: prospective randomized study. J Korean Surg Soc 2013;85:275-282.

4) de Araujo FB, Starling ES, Maricevich M, Tobias-Machado M. Single site and conventional totally extraperitoneal techniques for uncomplicated inguinal hernia repair: A comparative study. J Minim Access Surg 2014;10:197-201.
5) Yang GP, Tung KL. A comparative study of single incision versus conventional laparoscopic inguinal hernia repair. Hernia 2015; 19:401-405.

6) Wakasugi M, Masuzawa T, Tei M, et al. Single-incision totally extraperitoneal inguinal hernia repair: our initial 100 cases and comparison with conventional three-port laparoscopic totally $\mathrm{ex}^{-}$ traperitoneal inguinal hernia repair. Surg Today 2015;45:606-610.

7) Hamilton DF, Lane JV, Gaston P, et al. What determines patient satisfaction with surgery? A prospective cohort study of $4709 \mathrm{pa}^{-}$ tients following total joint replacement. BMJ Open 2013;3.

8) Pietrabissa A, Sbrana F, Morelli L, et al. Overcoming the challenges of single-incision cholecystectomy with robotic single-site technology. Arch Surg 2012;147:709-714.

9) Romanelli JR, Roshek TB, 3rd, Lynn DC, Earle DB. Single-port laparoscopic cholecystectomy: initial experience. Surg Endosc 2010;24:1374-1379.

10) Tai HC, Lin CD, Chung SD, Chueh SC, Tsai YC, Yang SS. A comparative study of standard versus laparoendoscopic singlesite surgery (LESS) totally extraperitoneal (TEP) inguinal hernia repair. Surg Endosc 2011;25:2879-2883.

11) Berger $P$. The journey to pain relief : a hands-on guide to breakthroughs in pain treatment. 1st ed. Alameda, CA: Hunter House; 2007. p.52.

12) Younger J, McCue R, Mackey S. Pain outcomes: a brief review of instruments and techniques. Curr Pain Headache Rep 2009;13:3943.

13) El-Oteify M, El-Dien HMS, Mubarak W. Sensory nerve endings in the human female umbilical skin: light and electron microscopic study. Egypt J Histol 2011;34:57-68. 